Portland State University

PDXScholar

9-1-2009

\title{
Influence of Illumination on Dark Current in Charge- Coupled Device Imagers
}

\author{
Ralf Widenhorn \\ Portland State University \\ Ines Hartwig \\ Justin Charles Dunlap \\ Portland State University \\ Erik Bodegom \\ Portland State University
}

Follow this and additional works at: https://pdxscholar.library.pdx.edu/phy_fac

Part of the Physics Commons

Let us know how access to this document benefits you.

\section{Citation Details}

Widenhorn R, Hartwig I, Dunlap JC, Bodegom E; Influence of illumination on dark current in chargecoupled device imagers. J. Electron. Imaging. 0001;18(3):033015-033015-8.

This Article is brought to you for free and open access. It has been accepted for inclusion in Physics Faculty Publications and Presentations by an authorized administrator of PDXScholar. Please contact us if we can make this document more accessible: pdxscholar@pdx.edu. 


\title{
Influence of illumination on dark current in charge-coupled device imagers
}

\author{
Ralf Widenhorn \\ Ines Hartwig \\ Justin C. Dunlap \\ Erik Bodegom \\ Portland State University \\ Department of Physics \\ Portland, Oregon 97207-0751 \\ E-mail: jdunlap@pdx.edu
}

\begin{abstract}
Thermal excitation of electrons is a major source of noise in charge-coupled-device (CCD) imagers. Those electrons are generated even in the absence of light, hence, the name dark current. Dark current is particularly important for long exposure times and elevated temperatures. The standard procedure to correct for dark current is to take several pictures under the same condition as the real image, except with the shutter closed. The resulting dark frame is later subtracted from the exposed image. We address the question of whether the dark current produced in an image taken with a closed shutter is identical to the dark current produced in an exposure in the presence of light. In our investigation, we illuminated two different $C C D$ chips with different intensities of light and measured the dark current generation. A surprising result of this study is that some pixels produce a different amount of dark current under illumination. Finally, we discuss the implication of this finding for dark frame image correction. (9) 2009 SPIE and IS\&T. [DOI: 10.1117/1.3222943]
\end{abstract}

\section{Introduction}

A major source of noise in charge-coupled-device (CCD) imagers is the generation of dark current. Even without the presence of light one finds that an image is not all black. White dots appear in the image and represent pixels with a high signal caused by impurities located in the silicon wafer. The dark signal for all pixels is caused by thermal excitation of electrons from the valence into the conduction band of silicon. A basic understanding of dark current in a $\mathrm{CCD}$ can be gained by analyzing the dark current generated in the p-n junction of a diode. ${ }^{1-5}$ It has been shown that the nonuniformity in the dark count is caused by deep-level impurities, such as $\mathrm{Ag}, \mathrm{Fe}$, or $\mathrm{Ni}^{6}{ }^{6-10}$ The fixed pattern of the dark count can be corrected by subtracting a so-called dark frame. A dark frame is generated by taking an image under the same condition as the light exposure (equal exposure time and temperature), with the difference that the shutter remains closed. To decrease the effects of dark current shot noise, often multiple dark frames are taken and

Paper 09048PRR received Apr. 13, 2009; revised manuscript received Jul. 21, 2009; accepted for publication Jul. 22, 2009; published online Sep. 8, 2009. This paper is a revision of a paper presented at the SPIE conference Sensors, Cameras, and Systems for Industrial/Scientific Applications IX, January 2008, San Jose, California. The paper presented there appears (unrefereed) in SPIE Proceedings Vol. 6816.

1017-9909/2009/18(3)/033015/8/\$25.00 @ 2009 SPIE and IS\&T. averaged to generate a so-called master frame. If one does not have the time to obtain a master dark frame for each imaging session, then one can also calibrate the dark current of a CCD chip. After the imager is calibrated for a certain temperature and exposure time range, one can compute a dark frame almost instantly. ${ }^{11}$

Every dark current subtraction assumes that the thermal generation of electrons is identical under illuminated and nonilluminated conditions. Hence, one assumes that the dark current generation is not affected by the presence of light or by the electrons that have already been optically excited and collected in the well.

In this study, we illuminated different CCDs with light to partially fill the well with electrons. We then compared the dark frame generated under the preilluminated condition to a conventional dark frame.

\section{Experimental Setup}

For our study, we used two different cameras: in the SBIG ST-8XE with a KAF-1602E CCD sensor and the Meade Pictor 416XT with a KAF-0400 CCD sensor. Both CCD chips are buried-channel frontside-illuminated CCDs. The KAF- $1602 \mathrm{E}$ is a $1530 \times 1020$ pixel array with an individual pixel size of $9 \times 9 \mu \mathrm{m}$. The KAF-0400 has the same individual pixel size, but only $768 \times 512$ pixels. Both cameras are frequently used by astronomers. We placed a lightemitting diode (LED) operated with a constant current supply at a distance of about $10-20 \mathrm{~cm}$ from the camera so that the whole chip was illuminated approximately uniformly.

In order to control the influx of light during an exposure an external shutter was mounted in front of the camera. The internal camera shutter was opened during all exposures. The external shutter was opened for $100-800 \mathrm{~ms}$, at the beginning of the exposure. Only for the short time that the external shutter is opened did light reach the CCD. After the external shutter was closed, the remaining exposure was done in the "dark." The light level was controlled by varying the opening time of the external shutter, the current through the LED, and the distance between camera and light source. Immediately following each light exposure, a regular dark frame with the same exposure time was taken, again with the internal shutter open and the light on; but 


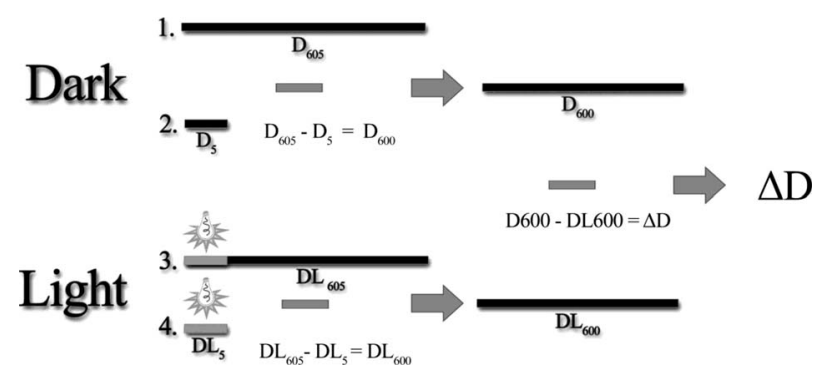

Fig. 1 Graphical representation for the process of comparing dark current in frames with and without light exposures.

this time the external shutter remained closed throughout the whole exposure. Figure 1 illustrates the process and the images taken for obtaining the change in dark count between frames with and without light exposure.

To measure the dark current over an integration time of $600 \mathrm{~s}$ with and without light, we took the following images:

1. $D L_{605}$ : Frame with the presence of light, $605 \mathrm{~s}$ exposure time, external shutter opened at the beginning of the exposure for 100-800 ms

2. $D L_{5}$ : Frame with the presence of light, $5 \mathrm{~s}$ exposure time, external shutter opened at the beginning of the exposure for $100-800 \mathrm{~ms}$

3. $D_{605}$ : Dark frame, $605 \mathrm{~s}$, closed external shutter

4. $D_{5}$ : Dark frame, $5 \mathrm{~s}$, closed external shutter

The light exposure at the beginning is the same for the $D L_{605}$ and the $D L_{5}$ frames and is subtracted out by taking the difference of the two exposures. Therefore, the dark current, $D L_{600}$, accumulated during $600 \mathrm{~s}$ after the image was initially exposed to light is given as

$D L_{600}=D L_{605}-D L_{5}$

The dark current, $D_{600}$, accumulated during $600 \mathrm{~s}$ while the camera was not exposed to light, is given as

$D_{600}=D_{605}-D_{5}$.

We will refer to this parameter as the normal dark count, because this is the count obtained in a regular dark frame.

To compare the dark count accumulated in $600 \mathrm{~s}$, one subtracts the dark count with light from the dark count without light:

$\Delta D=D_{600}-D L_{600}$.

For each exposure type and time, 20 and 25 images were taken for the Pictor 416XT and ST-8XE, respectively. The data presented here are the average values of those images. All data are expressed in counts, the number of electrons for each count can be obtained from the gain of each camera. For the SBIG ST-8XE, the gain is 2.3 counts $/ \mathrm{e}^{-}$, for the Meade Pictor 416XT the gain is 1.2 counts $/ \mathrm{e}^{-}$. The full well levels of the ST-8XE and the 416XT were 43,500 and 85,000 counts, respectively. All data presented here are below the saturation level of the chip.

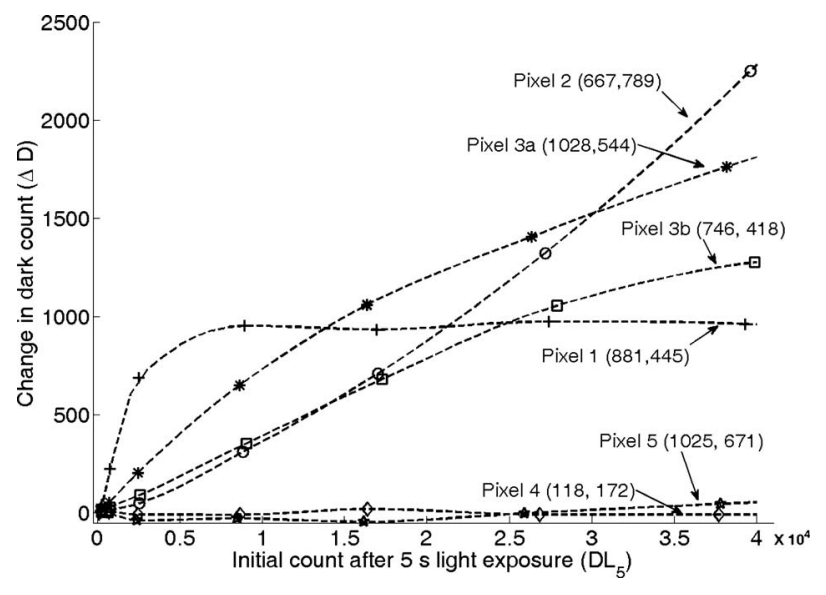

Fig. 2 Loss in dark count versus light level for six pixels for the ST-8XE. The dark count in the light exposures decreases with increasing light level. The numbers in the brackets are the $x$ and $y$ coordinates of the pixel.

\section{Results}

\subsection{SBIG ST-8XE}

One generally assumes that the dark current is independent of the light intensity. Under this assumption, the dark current for the $D L_{600}$ and the $D_{600}$ frames should be the same and $\Delta D$ approximately zero. However, this is not true for all pixels and light intensities. Figure 2 shows the change in the dark count, $\Delta D$, as a function of the average count of the initial 5-s of light exposure for six pixels. Independently of the initial illumination, pixels 4 and 5 do not show a significant change in the dark count. For pixels 2, 3a, and $3 \mathrm{~b}$, the dark count decreases with an increasing level of illumination.

The decrease in dark current slowly levels off for higher illumination levels. Pixel 1 also shows a decrease in dark count. Its decrease in dark count is roughly constant for initial light levels of 8000 counts or more.

The normal dark count for the pixels in Fig. 2 can be seen in Table 1 . At $288 \mathrm{~K}$, the average dark count across the chip without the presence of light, $D_{600}$, for a 600 -s exposure is 397 counts. Except for pixel 4, all pixels in Fig. 2 are hot pixels; that is the dark count is much larger than the average dark count of the image. As shown later, each pixel represents a group of pixels with a distinct behavior under illumination.

A two-dimensional distribution of the loss in dark count, $\Delta D$, versus the dark-count level, $D_{600}$, was created. The $\Delta D$ versus $D_{600}$ plane was divided into intervals of size $50 \times 50$ counts and then, for each pixel according to its dark-count values, the frequency for the corresponding interval is counted up by one. Figure 3 shows the resulting distributions for five different illumination levels. The shades of gray show how many pixels were in a specific interval. Because of the wide range of frequencies, the gray scale is represented as the logarithm of the frequency.

All five panels in Fig. 3 show a characteristic form that gets more defined with the increase of the initial illumination level. For a specific dark-count level, only very spe- 
Table 1 Normal dark count and change in dark count under illumination at the indicated counts.

\begin{tabular}{|c|c|c|c|c|c|c|}
\hline Pixel & Group & $D_{600}$ in counts & $\begin{array}{l}\Delta D / D_{600} \\
\text { at } D L_{5}=2,000 \\
\text { counts }(\%)\end{array}$ & $\begin{array}{l}\Delta D / D_{600} \\
\text { at } D L_{5}=8,500 \\
\text { counts }(\%)\end{array}$ & $\begin{array}{c}\Delta D / D_{600} \\
\text { at } D L_{5}=16,000 \\
\text { counts }(\%)\end{array}$ & $\begin{array}{c}\Delta D / D_{600} \\
\text { at } D L_{5}=26,000 \\
\text { counts }(\%)\end{array}$ \\
\hline 1 & 1 & 1,139 & 60.4 & 83.8 & 82.1 & 85.6 \\
\hline 2 & 2 & 5,956 & 0.8 & 5.2 & 11.9 & 22.2 \\
\hline $3 a$ & $3 a$ & 2,716 & 7.5 & 24.0 & 39.0 & 51.7 \\
\hline $3 b$ & $3 b$ & 2,846 & 3.2 & 12.4 & 23.9 & 37.1 \\
\hline 4 & Major peak & 184 & -4 & -5 & 10 & -5 \\
\hline 5 & Minor peak & 6,136 & -0.6 & -0.5 & -0.8 & 0.0 \\
\hline
\end{tabular}

cific ranges of values for the change in dark count were observed. On the basis of the decrease in dark count, we separated those pixels into different groups.

A large number of pixels have a small amount of dark current, and the light exposure does not change this behavior. These pixels are seen in Fig. 3 with close to zero dark current and small or no change in the dark current (main peak). Approximately 1.5 million or $97 \%$ of all pixels behave this way (see also Table 2). Pixel 4 in Fig. 2 is one example of a pixel located in the main peak. Its normal dark count is about 184 counts, and the change for all illumination levels is $<20$ counts. Pixel 5 in Fig. 2 is a pixel that is located in a second smaller peak of the distribution in Fig. 3. The $\sim 16,600$ pixels in this group have a large dark count, $D_{600}$, of about 6000 counts and show no change under illumination.

The greatest change in dark counts is exhibited by the pixels in group 1 . The change in dark count for those pixels is almost as high as their initial dark count. Hence, these pixels almost stopped generating dark current after being illuminated. One such pixel is pixel 1 in Fig. 2. Under "normal" conditions, without the presence of light, its dark count at $288 \mathrm{~K}$ and 600-s exposure time is 1139 counts. From Fig. 2, one observes that for an initial illumination level of roughly 8000 counts or more, the decrease is almost 1000 counts. This corresponds to only $\sim 14 \%$ of the dark count without the presence of light (see Table 1).

In Fig. 3, a straight line can be drawn to fit all the pixels in group 1. The slope of the straight-line fit is $>0.9$ for all five illumination levels depicted. As a result, pixels in group 1 have a decrease in dark count of $\sim 90 \%$ (e.g., $85.6 \%$ for pixel 1 in Fig. 2) of the dark count. Hence, those pixels show almost no dark count when flashed with an initial illumination level of 8000 counts or more (see Table 2).

Both groups $3 \mathrm{a}$ and $3 \mathrm{~b}$ show a decrease in dark level under illumination. Pixels $3 \mathrm{a}$ and $3 \mathrm{~b}$ are representative of these groups. From Fig. 2, one can see that the dark level decreases with increasing illumination over the whole range of illumination levels. The percentage decrease of the dark count is smaller than for group 1 . The decrease of the dark level for those pixels for the different illumination levels can again be found from the slope of a linear fit through the pixels of these groups. Pixels in group 3 a have

Table 2 Change in dark count for the different groups under different illumination levels. The number of pixels in each group was calculated using the constraints shown in Fig. 3 at 26,000 counts.

\begin{tabular}{lrcccc}
\hline \hline & & & $\begin{array}{c}\Delta D \text { at } \\
D L_{5}=8,500 \\
\text { counts }\end{array}$ & $\begin{array}{c}\Delta D \text { at } \\
D L_{5}=16,000 \\
\text { counts }\end{array}$ & $\begin{array}{c}\Delta D \text { at } \\
D L_{5}=26,000 \\
\text { counts }\end{array}$ \\
\hline Major peak & $1,519,003$ & $50-1,100$ & $-250-200$ & $-250-200$ & $-250-200$ \\
Minor peak & 16,628 & $5800-6200$ & $-250-200$ & $-250-200$ & $-250-200$ \\
Group 1 & 3638 & $1000-6000$ & $90 \%$ of $D_{600}$ & $90 \%$ of $D_{600}$ & $90 \%$ of $D_{600}$ \\
Group 2 & 757 & $5500-6600$ & $250-3250$ & $250-3250$ & $250-3250$ \\
Group 3a & 1347 & $1750-5450$ & $25 \%$ of $D_{600}$ & $40 \%$ of $D_{600}$ & $60 \%$ of $D_{600}$ \\
Group 3b & 1669 & $2350-5400$ & $15 \%$ of $D_{600}$ & $25 \%$ of $D_{600}$ & $50 \%$ of $D_{600}$ \\
Other & 17,558 & xxx & xxx & xxx & xxx \\
\hline \hline
\end{tabular}




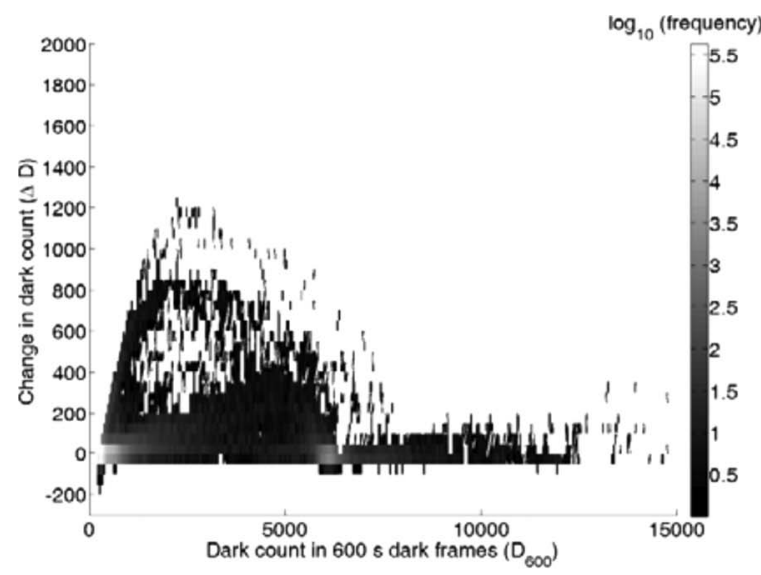

(a)

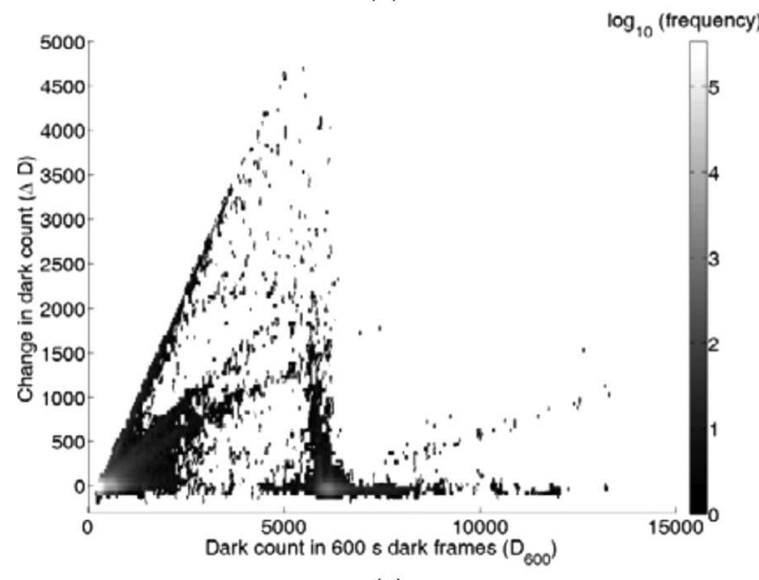

(c)

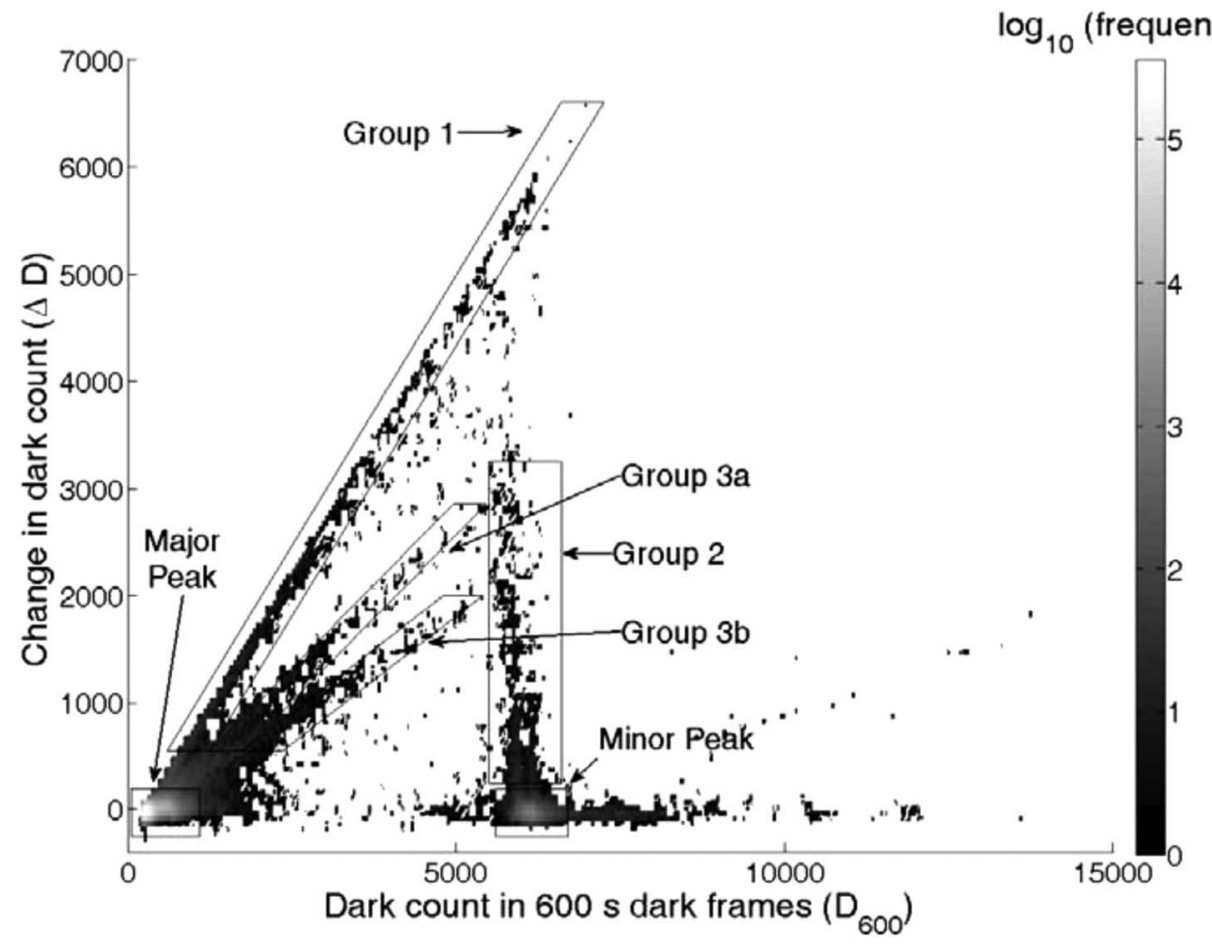

(e)

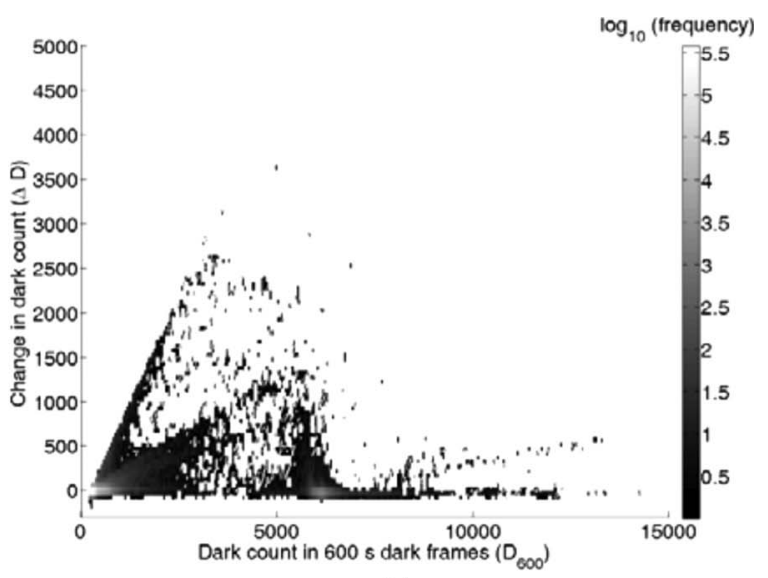

(b)

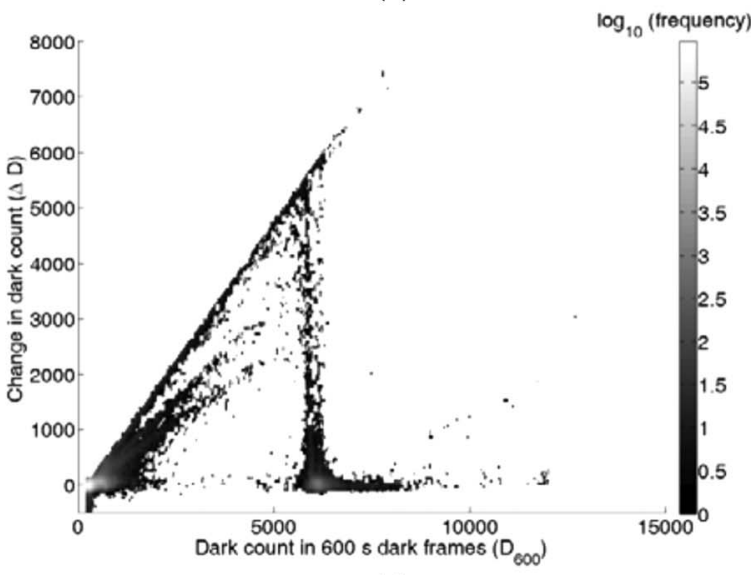

(d)

$g_{10}$ (frequency)

Fig. 3 Distribution of the change in dark count, $\Delta D$, at $288 \mathrm{~K}$ due to illumination for the SBIG ST-8XE for five different light levels. The average initial counts due to the light exposure were approximately: (a) 2,500 , (b) 8,500 , (c) 16,000 , (d) 37,000 , and (e) 26,000 counts. 
a decrease of $\sim 25 \%$ at 8600 counts illumination [Fig. 3(b)] $40 \%$ at 16,000 counts illumination [Fig. 3(c)], and $60 \%$ at 26,000 counts illumination [Fig. 3(e)]. Hence, the dark current level is from $75 \%$ of the normal level at 8600 count illumination to $40 \%$ of the normal level at 26,000 count illumination.

Group $3 \mathrm{~b}$ has the same characteristics as group 3a, with the percentage decrease being slightly smaller. At 8600 counts illumination, the dark count is decreased by $15 \%$; at 16,000 counts illumination, it is decreased by $25 \%$; and at 26,000 counts illumination, it is decreased by $50 \%$.

Finally, group 2 is formed by pixels that have the same normal dark count as the pixels grouped in the minor peak. However, under illumination their dark current decreases. As with groups $3 \mathrm{a}$ and $3 \mathrm{~b}$, the dark count decreases with increasing illumination. Pixel 2 in Fig. 2 belongs to this group. The percentage change in dark count for all pixels in Fig. 2 can be seen in Table 1.

A summary of the number of pixels in the individual groups and their change in dark count under different illumination levels can be seen in Table 2. Of the pixels outside these groups, 3467 pixels have no change in dark count under illumination. Furthermore, there are a small number of pixels that have a dark count larger than in the minor peak and a slight decrease with illumination. We found that there is a significantly larger number of those pixels in the KAF-0400 chip and will look at them in more detail in Sec. 3.2. There are also some pixels that show the opposite effect and have an increase in dark current with illumination. Most of these lie below the main peak, and we found that these pixels have a very hot neighboring pixel. After the hot pixel reaches saturation, electrons can spill over giving those pixels an increasing amount of dark current with an increase in illumination.

\subsection{Meade Pictor 416XT}

The second CCD investigated in this study was the Kodak KAF-0400 housed in the Meade Pictor 416XT. For the Pictor 416XT camera, data at $278 \mathrm{~K}$ were taken. The experimental setup and analysis were done in the same way as for the SBIG camera.

Figure 4 shows the distribution for the change in dark count for three different illumination levels. The images were flashed with light that caused an average count of approximately 12,500 [Fig. 4(a)], 25,500 [Fig. 4(b)], and 42,100 counts [Fig. 4(c)].

One finds many similarities and some differences in the characteristic for the two chips. For the Meade, instead of the two peaks, one finds another minor peak. The majority of pixels are regular pixels with very little dark current located in the major peak. Their average dark count at $278 \mathrm{~K}$ and $600 \mathrm{~s}$ exposure time is $\sim 500$ counts. There are two groups of pixels with a dark count of $\sim 5700$ counts (minor peak 1) and 10,900 counts (minor peak 2), respectively. It is interesting to note that pixels in all three peaks have a dark count of $\sim 250$ counts more when illuminated to light. The normal dark count of the pixels in minor peak 2 is about twice the dark count of the minor peak 1 pixels, making it likely that the same impurity occurs twice within that group.

Group 1a for the 416XT is analogous to group 1 for the ST-8XE. A straight line drawn through the pixels of group 1a has a slope of $\sim 1$. Hence, pixels in this group generate almost no dark count when illuminated. Of course, to this one needs to add the global positive offset of about 250 counts. One can also see a number of pixels with a similar slope in the $D_{600}$ versus $\Delta D$ graph emerge from the first minor peak. Because of this similarity, we will call those pixels group $1 \mathrm{~b}$. There are 1376 pixels, $<10 \%$ of group $1 \mathrm{a}$, in group $1 \mathrm{~b}$ (see Table 3 ). This is still a sizeable number, and one generally notes that there is a much larger number of pixels outside the major and minor peak groups for the Meade. Only $\sim 80 \%$ of the pixels are in the major peak for the Meade camera, for the SBIG camera $\sim 97 \%$ are in the major peak. One can explain pixels in group $1 \mathrm{~b}$ by assuming the presence of a minor peak 1 plus a group 1a-type impurity in the same pixel.

Using the same reasoning for group 2, one can conclude that group $2 \mathrm{~b}$ pixels contain a minor peak 1 plus a group $2 a-t y p e$ impurity. Again, the number of pixels in groups $2 \mathrm{a}$ and $2 b$ is much larger than the number of pixels in Group 2 of the SBIG camera. Only $0.05 \%$ of all pixels on the chip are in group 2, while the percentage is much larger for the corresponding groups $2 \mathrm{a}$ and $2 \mathrm{~b}$ with 1.8 and $0.3 \%$, respectively (see Tables 2 and 3). Groups $3 \mathrm{a}$ and $3 \mathrm{~b}$ for the SBIG camera do not a have an equivalent in the Meade camera. Instead, there is a larger number of pixels (group 3 with 1751 pixels) with a normal dark count larger than the first minor peak and a significant loss in dark count. The slope of a linear fit through group 3 pixels in Fig. 4 at 42,100 counts is given by $\sim 0.3$. It appears likely that those pixels are the result of a minor peak 1 plus another secondary impurity. One can further note from Fig. 3 that the SBIG camera seems to contain a similar type of pixel as well. However, as with the rest of the chip, the number of pixels with impurities was smaller and we did not assign them in a separate group. Groups $2 \mathrm{~b}$ and 3 overlap. We counted 427 pixels twice as being both in groups $2 \mathrm{~b}$ and 3 also 9931 pixels are not in any of the groups, with 5045 of those at the baseline with an increase in the dark count of $\sim 250$ counts.

As can be seen from Fig. 5, the pixels on the KAF-0400 imager respond to increasing illumination in a similar manner as do the corresponding groups on the KAF-1602E sensor shown in Fig. 2. The pixels lying in the peaks, in this case pixel 4 lies in the main peak, pixel 5 lies in minor peak 1 , and pixel 6 lies in minor peak 2, do not show a significant change with changing illumination level. Pixels $1 \mathrm{a}, 1 \mathrm{~b}$, $2 \mathrm{a}, 2 \mathrm{~b}$, and 3 all show a decreasing amount of dark count with increasing levels of illumination, and all lie within their correspondingly named region in Fig. 4(c). For pixels $1 \mathrm{a}$ and $1 \mathrm{~b}$, the amount of dark current levels off for higher illumination levels. The decrease in dark count is roughly constant for pixel $1 \mathrm{~b}$ after $\sim 20,000$ counts and is roughly constant for pixel 1a after 35,000 counts.

\section{Discussion}

We found that results are independent of the wavelength, $\lambda$, of the illuminating light and therefore independent of the location where photoelectrons are generated. The data for a red LED with $\lambda=650 \mathrm{~nm}$ and a penetration depth in silicon of $3 \mu \mathrm{m}$ yielded the same characteristics as a blue LED with $\lambda=430 \mathrm{~nm}$ and a penetration depth of only $300 \mathrm{~nm}$.

The distributions for the same light level at different 


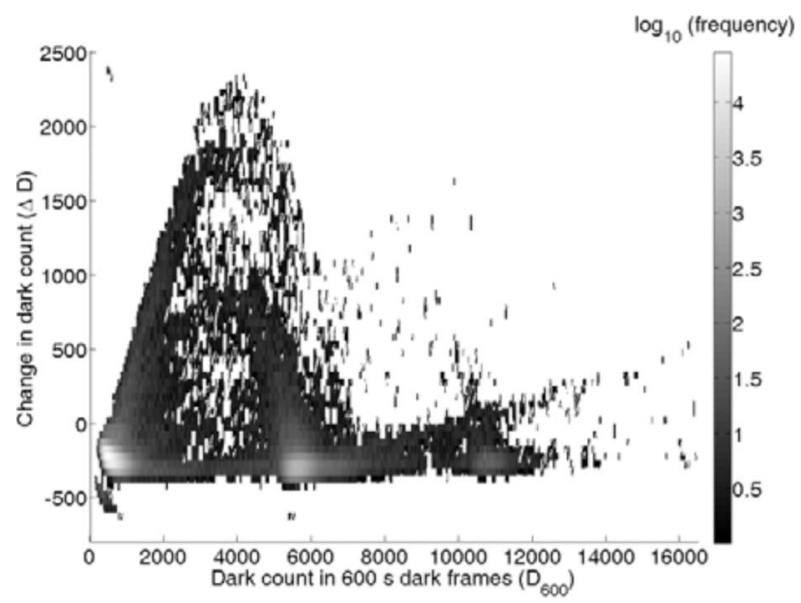

(a)

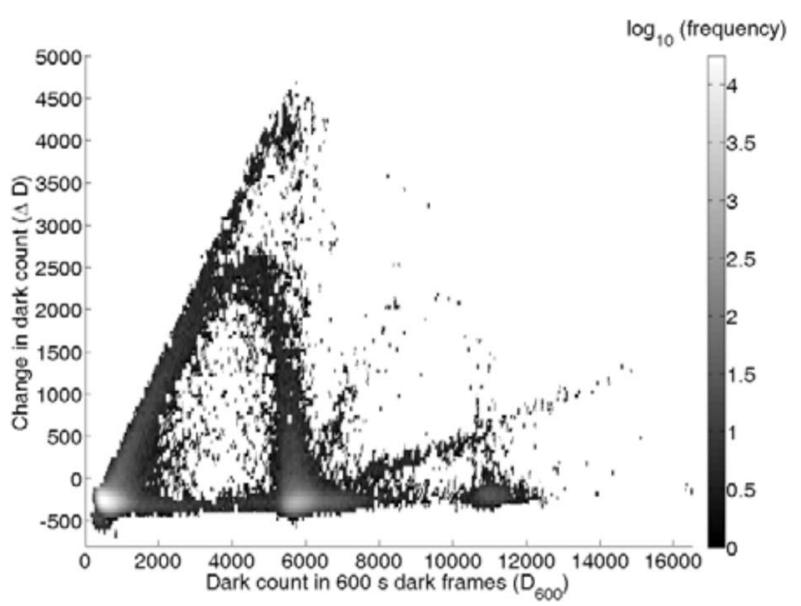

(b)

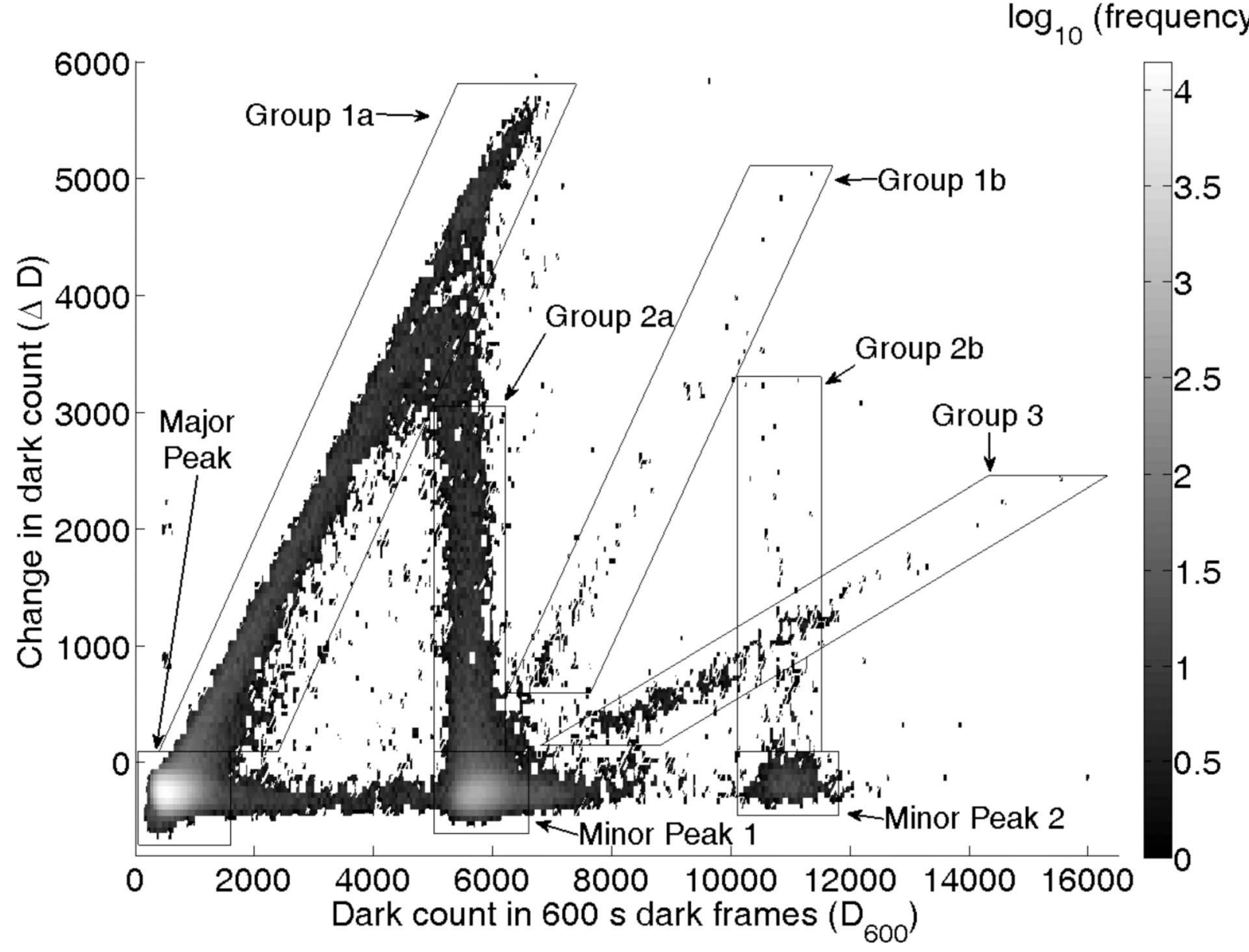

(c)

Fig. 4 Distribution of the change in dark count at $278 \mathrm{~K}$ due to illumination for the Pictor 416XT for three different light levels. The average initial counts due to the light exposure were approximately: (a) 12,500 , (b) 25,500 , and (c) 42,100 counts.

temperatures were measured, and the same characteristics were found at those temperatures as well. It was further noted that the pixels of all groups were found to be uniformly distributed over the whole area of the chip.

Most pixels for the SBIG camera fall in the two peaks and do not show a significant change in dark current. On the other hand, for larger intensities a majority of pixels for the Meade camera show an actual increase in dark count. Hence, a regular dark frame would predict a dark count that is too small. Our data indicate that this is a global phenomenon across the whole chip, resulting in a constant offset. What makes an accurate dark-count correction more prob- 
Table 3 Change in dark count for the different groups under different illumination levels. The parameter, Off $=250$ counts, represents the dark count increase of a regular pixel under illumination. The dark count of a pixel in the first minor peak is given approximately as $D_{\mathrm{MP} 1}=5700$ counts: The number of pixels in each group was calculated using the constraints shown in Fig. 4 at 42,100 counts.

\begin{tabular}{|c|c|c|c|c|}
\hline Group Name & No. of Pixels & $D_{600}$ in counts & $\begin{array}{c}\Delta D \text { at } \\
D L_{5}=25,500 \text { counts }\end{array}$ & $\begin{array}{c}\Delta D \text { at } \\
D L_{5}=42,100 \text { counts }\end{array}$ \\
\hline Major peak & 322,051 & $50-1600$ & $-700-100$ & $-700-100$ \\
\hline Minor peak 1 & 30,818 & $5000-6600$ & $-600-100$ & $-600-100$ \\
\hline Minor peak 2 & 1550 & $10,100-11,800$ & $-450-100$ & $-450-100$ \\
\hline Group 1a & 16,332 & $400-7400$ & $1 \times D_{600}$-Off & $1 \times D_{600}$-Off \\
\hline Group 1b & 1376 & $6200-11,700$ & $1 \times\left(D_{600}-D_{\mathrm{MP} 1}\right)-\mathrm{Off}$ & $1 \times\left(D_{600}-D_{\mathrm{MP} 1}\right)-\mathrm{Off}$ \\
\hline Group 2a & 6962 & $5000-6200$ & $100-3050$ & $100-3050$ \\
\hline Group 2b & 1250 & $10,100-11,500$ & $100-3300$ & $100-3300$ \\
\hline Group 3 & 1751 & $7600-16,300$ & $0.2 \times\left(D_{600}-D_{\mathrm{MP} 1}\right)-\mathrm{Off}$ & $0.3 \times\left(D_{600}-D_{\mathrm{MP} 1}\right)-\mathrm{Off}$ \\
\hline Group $2 b$ and 3 & 427 & $x x x$ & $x x x$ & $x x x$ \\
\hline Other & 9931 & $x x x$ & $x x x$ & $x x x$ \\
\hline
\end{tabular}

lematic is the fact that some pixels will show a decrease in dark count. Under large-enough illumination, pixels in group 1 almost stop generating dark electrons. Of course, in an actual exposure the photoexcitation of electrons due to the incoming light does not happen all at the beginning of the exposure. Assuming a constant intensity of light, pixels in group 1 might generate dark current at a normal rate at the beginning of the exposure and then transforms to a non-dark-count-generating state toward the end of the exposure. Our analysis can help to predict how much dark count is generated during different stages of the exposure. This, however, assumes that the light intensity is constant and does not vary with time. If one does not have enough

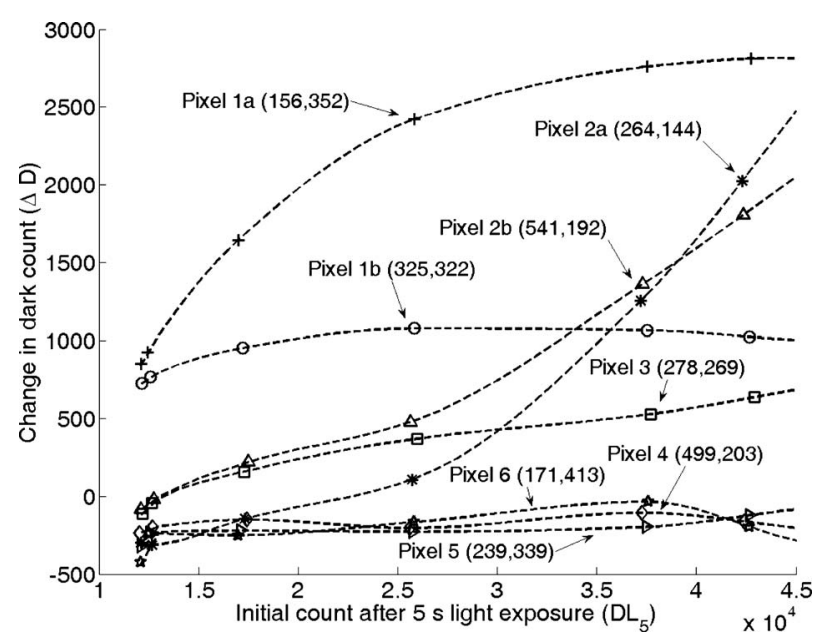

Fig. 5 Loss in dark count versus light level for eight pixels for the Pictor 416XT. The numbers in the brackets are the $x$ and $y$ coordinates of the pixel. knowledge of possible changes in the light intensity during the exposure, then an accurate prediction of the dark count of pixels in group 1 is virtually impossible.

The difficulties in correcting dark current accurately for pixels in groups 2 and 3 are similar to those for pixels in group 1. There are roughly 21,000 pixels, or $1.4 \%$ of the total pixels, for the SBIG camera $(T=288 \mathrm{~K}, 600$-s exposure time), where the dark current differs by $>200$ counts for an exposure with an initial illumination level of 26,000 counts. The same pixels would show a different dark count to a varying degree under all illumination conditions. Ignoring the global increase of dark current under illumination, there are roughly 28,000 pixels, or $7.1 \%$ of the total pixels, in groups 1-3 that have a dark count that is strongly illumination level dependent for the Meade camera.

Finally, because dark current is strongly linked to impurities in the CCD, our results can lead to a new understanding of the nature of those impurities. Impurities are generally characterized by the dark current they produce at a particular exposure time and temperature. By varying the temperature, one can also find the activation energy of a particular impurity. For example, the activation energy can be used to distinguish between diffusion current generated in the field-free region or depletion current from within the potential well. ${ }^{6}$ But the nature of impurities also differs by their behavior under illumination. A pixel in the minor peak and a group 2 pixel show the same normal dark count. They would be characterized as being hot and containing one particular impurity. However, our data clearly show that, while they may have the same impurity, the nature of the defect in these pixels differs. Possible explanations are that the location of the impurity within the pixel could be the cause of the various dark current responses to illumination, or that traps, for example, located at the surface, could 
be filling with photogenerated electrons. Further work in the study of the time-dependence of dark current in these pixels could yield additional information on the nature of impurities in a sensor.

\section{Conclusion}

Dark current is generally assumed to be independent of the light level of the exposure. Differences between the actual number of dark electrons in the real exposure and a dark frame are generally assumed to be due to statistical noise. However, our study shows clearly that certain pixels show a systematic difference in their dark count under illumination.

The change in dark count $\Delta D$ in $600 \mathrm{~s}$ cannot assume any value for a given level of light exposure-we found that only specific areas in the $\Delta D$ versus $D_{600}$ plane were observed. We were able to group the behavior of pixels according to this discrete change. Pixels in each group may contain one or more specific types of impurities.

We hope this study will help contribute to a better understanding of the nature of dark current and an even further improvement of CCD image quality. More immediately, the results help to accurately correct for dark current and in the computation of dark frames.

\section{References}

1. A. S. Grove, Physics and Technology of Semiconductor Devices, Wiley, Hoboken, NJ (1967).

2. C. T. Sah, R. N. Noyce, and W. Shockley "Carrier generation and recombination in p-n junction and p-n junction characteristics," Proc. IRE, 45, 1228-1248 (1957).

3. R. N. Hall, "Electron-hole recombination in germanium," Phys. Rev 87, 387-388 (1952)

4. W. Shockley and W. T. Read, "Statistics of the recombination of holes and electrons," Phys. Rev. 87, 835-842 (1952)

5. S. M. Sze, Physics of Semiconductor Devices, 2nd ed., Wiley, Hoboken, NJ (1981)

6. R. Widenhorn, M. M. Blouke, A. Weber, A. Rest, and E. Bodegom, "Temperature dependence of dark current in a CCD," Proc. SPIE 4669, 193-201 (2002)

7. R. D. McGrath, J. Doty, G. Lupino, G. Ricker, and J. Vallerga, "Counting of deep-level traps using a charge-coupled device," IEEE Trans. Electron Devices 34, 2555-2557 (1987)

8. W. C. McColgin, J. P. Lavine, J. Kyan, D. N. Nichols, and C. V. Stancampiano, "Dark current quantization in CCD image sensors," in Tech. Dig. - Int. Electron Devices Meet., 113-116 (Dec. 1992).

9. W. C. McColgin, J. P. Lavine, and C. V. Stancampiano, "Probing metal defects in CCD image sensors," Mater. Res. Soc. Symp. Proc. 378, 713-724 (1995).

10. W. C. McColgin, J. P. Lavine, C. V. Stancampiano, and J. B. Russell, "Deep-level traps in CCD image sensors," Mater. Res. Soc. Symp. Proc. 510, 475-480 (1998).
11. R. Widenhorn, A. Rest, M. M. Blouke, R. L. Berry, and E. Bodegom, "Computation of dark frames in digital imagers," Proc. SPIE $\mathbf{6 5 0 1}$ 650103 (2007)

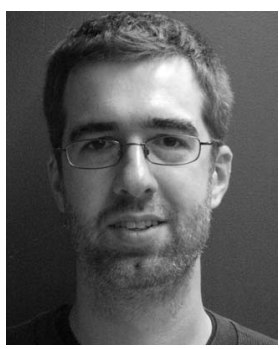

Ralf Widenhorn received his Vordiplom in physics from University of Konstanz in 1997, his MS in physics in 2000, and his $\mathrm{PhD}$ in environmental sciences and resources/physics in 2005, both from Portland State University. He has numerous published papers on digital imagers and is currently a researcher and professor at Portland State University. Some of his work is available in the book, Charge-Coupled Devices-Performance and Dark Noise Characteristics.

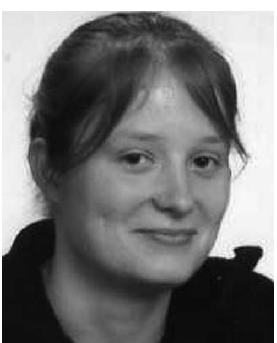

Ines Hartwig received her BS and MS in computational science from Chemnitz University of Technology in 2005 and 2007. During a one-year stay in 2005/2006, she obtained her MS in physics from Portland State University. She is currently completing her PhD on the dynamics of nonlinear disordered systems at Chemnitz University.

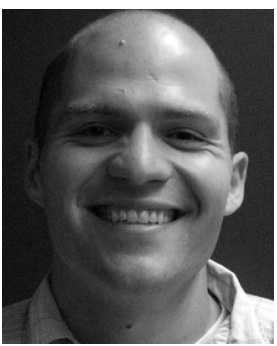

Justin C. Dunlap received his BA in physics and astrophysics from the University of California, Berkeley, in 2002. He received his MS in physics from Portland State University in 2009. He is currently a graduate student at Portland State University continuing to research dark current and digita imagers.

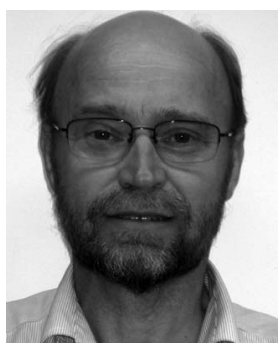

Erik Bodegom completed his engineering degree at Technical University Delft, Netherlands, and his PhD (1982) at Catholic University of America in Washington, DC Since 1985, he has been with Portland State University, where he is professor and chair of the Department of Physics. His pedagogical activities have included the writing of a set astronomical exercises, the development of a resistance probe for use in schools, and the development of software to remotely control observatories. He has also served as president of the Oregon Academy of Science. His research activities have ranged from low-temperature physics to statistical mechanics and solid-state physics. Over the last decade, his work has focused on the use and understanding of digital imagers. 